\title{
PROPAGACION VEGETATIVA DE TEPA (Laurelia philippiana), LINGUE (Persea lingue) Y MAÑIO (Podocarpus saligna) A PARTIR DE ESTACAS
}

Rómulo Santelices (*)

\section{RESUMEN}

En este estudio se analiza la posibilidad de propagar vegetativamente las especies nativas Tepa (Laurelia philippiana), Lingue (Persea lingue) y Mañío (Podocarpus saligna), mediante el arraigamiento de estacas colectadas en primavera.

Las estacas fueron tratadas con ácido indolbutíico (A/B) y se aplicaron tres diferentes niveles de temperatura en la base de éstas. Se las mantuvo en invernadero durante 10 meses y al final de este período se evaluó la supervivencia y la emisión de raíces. Posteriormente, se las transplantó al vivero y después de un año se evaluó la supervivencia y el crecimiento en altura y diámetro.

Se constató que las especies en estudio pueden ser propagadas vegetativamente por este método y que de los niveles de temperatura probados los más adecuados son $21^{\circ} \mathrm{C}$ para Tepa, $18-24^{\circ} \mathrm{C}$ para Lingue y $24^{\circ} \mathrm{C}$ para Mañio.

Palabras clave: Propagación vegetativa. Laurelia philippiana.

Persea lingue. Podocarpus saligna.

\section{ABSTRACT}

The possibility of vegetative propagation of Tepa (Laurelia philippiana), Lingue (Persea lingue) and Mañio (Podocarpus saligna), from rooting of cuttings collected in spring time is analized in this paper.

The cuttings were treated with indolebutyric acid (IBA) and three different temperature levels were applied. Cuttings stayed 10 months in a greenhouse and later they were moved to the nursery for one year. At the end of both, the greenhouse and the nursery periods, the cuttings were controled.

The species studied can be propagated by this methods and best results were obtained under a temperature of $21^{\circ} \mathrm{C}$ for Tepa, $18-24^{\circ} \mathrm{C}$ for Lingue and $24^{\circ} \mathrm{C}$ for Mañio.

Keywords: Vegetative propagation. Laurelia philippiana. Persea lingue. Podocarpus saligna. 


\section{INTRODUCCION}

Tepa (Laurelia philippiana), Lingue (Persea lingue) y Mañío (Podocarpus saligna), son especies del bosque nativo chileno, consideradas tolerantes, que se pueden encontrar en diferentes asociaciones con otras, principalmente del género Nothofagus. Sus maderas son de gran valor, destacando su uso para la fabricación de chapas, tableros contrachapados y mueblería.

Debido a la gran demanda por sus maderas, los bosques con estas especies han sido sometidos a reiteradas explotaciones. Como se ha tendido a extraer lo mejor, sin un criterio silvícola, estos bosques se han empobrecido, tanto en la frecuencia como en la calidad de las especies remanentes.

Para revertir esta situación es urgente enriquecer estos bosques a través de plantaciones que servirán como complemento a la regeneración natural.

Una de las formas de reproducción es la propagación vegetativa. Esta se basa en la omnipotencia celular, es decir, las plantas son capaces de construir un organismo completo a partir de unas pocas células (cultivo meristemático) o en caso extremo a partir de una sola célula. Este método es una herramienta útil para regenerar algunas especies nativas de difícil propagación generativa.

Por ejemplo para Tepa, se lograron resultados preliminares que ya muestran la eficacia del método. Estacas de esta especie colectadas en otoño, tratadas en sus bases con temperaturas entre 20 y $25^{\circ} \mathrm{C}$ durante 6 meses en invernadero y posteriormente transplantadas a vivero por 1,5 años, desarrollaron una buena producción de raices y registraron altos niveles de sobrevivencia y crecimiento (Santelices, 1990).

En el presente trabajo se amplía la investigación probando con estacas colectadas en primavera y con un mayor número de niveles de temperatura. El estudio se complementa con las especies lingue y mañio.

\section{MATERIAL Y METODO}

\section{Diseño Experimental}

El ensayo se planteó con un diseño estadístico completamente al azar, con tres tratamientos, 3 réplicas y 24 estacas por parcela. 
Los niveles de temperatura en la base de las estacas (T) fueron los siguientes:

$\mathrm{T} 1: 18^{\circ} \mathrm{C}$

$\mathrm{T} 2: 21^{\circ} \mathrm{C}$

$\mathrm{T} 3: 24^{\circ} \mathrm{C}$

\section{Estacas}

Las estacas de tepa utilizadas en el ensayo provienen del sector "Depósito" en la ribera sur-este del lago Pirehueico. Las estacas de lingue y mañío se colectaron en el área de Jauja, Precordillera Andina. El material de tepa y lingue se eligió de rebrotes de tocón. Para mañío no se encontraron tocones rebrotados, razón por la cual las estacas se seleccionaron de árboles jóvenes regenerados por monte alto bajo dosel, de una altura media de $80 \mathrm{~cm}$ (Cuadro $\mathrm{N}^{\circ} 1$ ).

\section{Cuadro $\mathrm{N}^{\circ} 1$}

ORIGEN DEL MATERIAL ENSAYADO

\begin{tabular}{|l|l|l|l|}
\hline \multirow{2}{*}{ Antecedentes } & \multicolumn{3}{|c|}{ Especie } \\
\cline { 2 - 4 } & Tepa & Lingue & Mañio \\
\hline Procedencia & Depósito & Jauja & Jauja \\
LongitudW & $71^{\circ} 53^{\prime}$ & $72^{\circ} 01^{\prime}$ & $72^{\circ} 01^{\prime}$ \\
LatitudS & $39^{\circ} 53^{\prime}$ & $38^{\circ} 06^{\prime}$ & $38^{\circ} 06^{\prime}$ \\
Altitud(msnm) & 630 & 790 & 600 \\
Edadaproximada(años) & 80 & $20-35$ & $3-9$ \\
Exposición & Enplano & Enplano & En plano \\
Tipoderebrote & Basefuste & Tocón & Regeneración \\
Edadderebrote(años) & $1-2$ & 0,33 & $3-9$ \\
Arboles & 6 & 5 & 5 \\
\hline
\end{tabular}

\section{Instalación delEnsayo}

La metodología seguida en este trabajo se basa en los antecedentes sobre la propagación de estacas proporcionados por Silva (1968), Krussmann (1981) y Santelices (1990). 
El ensayo se llevó a cabo en el invernadero y vivero del Centro Experimental Escuadrón de Forestal Mininco, ubicado $17 \mathrm{~km}$ al sur de la ciudad de Concepción.

La cosecha de material se realizó de madrugada en los primeros días del mes de Noviembre de 1988. Este se mantuvo siempre húmedo y se transportó ese mismo dia al invernadero. Durante la noche se dejó con su base en agua y en completa obscuridad hasta el día siguiente, cuando se procedió a cortar las estacas. El primer corte se realizó $15 \mathrm{~cm}$ por debajo del brote apical.

Las estacas seleccionadas presentaron al menos 2 hojas y 3 brotes, de los cuales uno se localizó en sus bases. Los cortes se realizaron en un ángulo de $45^{\circ}$ bajo agua fría, manteniéndose las estacas dentro de ésta.

El material se sumergió durante 15 minutos en una solución de AIB (1.000 ppm) mezclado con alcohol etílico $\left(\mathrm{CH}_{3} \mathrm{CH}_{2} \mathrm{OH}\right)$, tomándose las precauciones necesarias para evitar la degradación del ácido por efecto de la luz.

La temperatura en la base de las estacas fue controlada en una piscina con agua temperada. Esta se construyó con madera y como aislante se usó poliestireno. La parte interior se cubrió con polietileno. Cada nivel de temperatura fue regulado con termocalentadores eléctricos para acuarios.

A $10 \mathrm{~cm}$ sobre el nivel del agua se instaló un fina red sobre la cual se depositó el sustrato. Así se garantizó una óptima circulación de agua y oxígeno. El sustrato consistió en aserrín de Pinus radiata. Este se hirvió en agua por lo menos durante media hora para eliminar toxinas y hongos.

Las estacas se insertaron en las camas calientes de arraigamiento hasta una profundidad de $7 \mathrm{~cm}$. Se mantuvieron bajo la luz de un tubo fluorescente (40 W) por 9,5 horas cada día.

Diariamente y a cada hora se registró la temperatura ambiental y de las camas de arraigamiento. Las hojas de las estacas se mantuvieron húmedas por el uso de nebulizadores, no pasando la temperatura ambiental de los $30^{\circ} \mathrm{C}$. Como medida preventiva se aplicó un fungicida sistémico y abono foliar a las hojas.

Las estacas permanecieron en las camas de arraigamiento del invernadero durante 10 meses, evaluándose finalmente esta etapa. Posteriormente fueron transplantadas al vivero, donde se mantuvieron cubiertas con malla Raschel de 50 $\%$ de cobertura para darles mayor protección.

Después de 1 año en el vivero, se controló la sobrevivencia y crecimiento de las estacas.

Durante el tiempo en el cual las estacas permanecieron en el vivero fueron sometidas a una poda horizontal de raíces, al término del período vegetativo y a una profundidad aproximada de $12 \mathrm{~cm}$. Además, periódicamente se realizaron controles fitosanitarios. 


\section{RESULTADOS Y DISCUSION}

A continuación se presentan los resultados del desarrollo de las estacas obtenido en la cama caliente de arraigamiento en el invernadero y en el vivero después de su transplante a las platabandas.

Después de permanecer 10 meses en estas camas de arraigamiento, se evaluó el desarrollo radicular de las estacas. La cantidad y longitud media de las raices producidas por estaca se presentan en el Cuadro $\mathrm{N}^{\circ} 2$

\section{Cuadro $N^{\circ} 2$}

PRODUCCION MEDIA DE RAICES Y SOBREVIVENCIA EN FUNCION DE LA TEMPERATURA DEL SUSTRATO

\begin{tabular}{|c|c|c|c|c|}
\hline \multirow[t]{2}{*}{ Especie } & \multirow{2}{*}{$\begin{array}{c}\text { Temperatura } \\
\text { Sustrato } \\
\left({ }^{\circ} \mathrm{C}\right)\end{array}$} & \multicolumn{2}{|c|}{ Prod. Media Raicespor Estaca (1) } & \multirow[t]{2}{*}{ Supervivencia (1) } \\
\hline & & $\begin{array}{c}\text { Cantidad } \\
\left(\mathrm{N}^{\circ}\right)\end{array}$ & $\begin{array}{l}\text { Longitud } \\
\text { (cm) }\end{array}$ & \\
\hline Tepa & $\begin{array}{l}18 \\
21 \\
24\end{array}$ & $\begin{array}{r}16,1 \mathrm{a} \\
16,8 \mathrm{a} \\
6,2 \mathrm{~b}\end{array}$ & $\begin{array}{l}3,9 a b \\
5,6 a \\
2,6 b\end{array}$ & $\begin{array}{l}99 a \\
92 a \\
72 b\end{array}$ \\
\hline Lingue & $\begin{array}{l}18 \\
21 \\
24\end{array}$ & $\begin{array}{l}0,6 a \\
0,9 a \\
2,5 a\end{array}$ & $\begin{array}{l}0,4 a \\
1,0 a \\
1,5 a\end{array}$ & $\begin{array}{l}35 a \\
29 a \\
35 a\end{array}$ \\
\hline Mañio & $\begin{array}{l}18 \\
21 \\
24\end{array}$ & $\begin{array}{l}6,2 \mathrm{~b} \\
5,1 \mathrm{~b} \\
7,5 \mathrm{a}\end{array}$ & $\begin{array}{l}1,5 b \\
2,9 b \\
6,7 a\end{array}$ & $\begin{array}{l}61 a \\
43 a \\
26 b\end{array}$ \\
\hline
\end{tabular}

(1) a, b, c: Valores medios señalados con letras minúsculas distintas se diferencian entre ellos a un nivel de confianza del $95 \%$.

Para todas las especies estudiadas se logró inducir el desarrollo de raíces. $\mathrm{Pa-}$ ra tepa, el mayor número de raíces y la más alta supervivencia se obtuvo con 18 y $21^{\circ} \mathrm{C}$, mientras que la mayor longitud de éstas con $21^{\circ} \mathrm{C}$. Con $24^{\circ} \mathrm{C}$ se consiguió un moderado desarrollo radicular y sobrevivencia. Este último resultado difiere del presentado por Santelices (1990), quién con $25^{\circ} \mathrm{C}$ obtuvo una alta producción de raices y sobrevivencia con estacas colectadas en otoño. Esta diferencia podría estar influenciada por la época de cosecha de las estacas. Es sabido que cada especie responde en forma diferente a los tratamientos que inducen la formación de raíces de las estacas. Uno de estos factores es la época de cosecha del mate- 
rial. Es posible que estacas de tepa colectadas en primavera deban tratarse en forma diferente que aquellas obtenidas en otoño.

Para lingue no se encontraron diferencias significativas entre los tratamientos probados. Sin embargo, a medida que se aumentó la temperatura, se observó una tendencia a una mayor producción de raíces. Para todos los niveles de temperatura se obtuvo un baja sobrevivencia, que varió entre 29 y $35 \%$.

Para mañío con $24^{\circ} \mathrm{C}$ se consiguió la más alta producción de raíces, en términos de cantidad y longitud de éstas. Con una mayor temperatura la sobrevivencia tendió a disminuir.

Para tepa el mejor desarrollo radicular se obtuvo entre los 18 y $21^{\circ} \mathrm{C}$. Para lingue fue entre los 18 y $24^{\circ} \mathrm{C}$, mientras que para mañío éste se consiguió con $24^{\circ} \mathrm{C}$. La supervivencia baja para tepa y mañío a medida que aumenta la temperatura, en tanto que para el caso del lingue no se aprecia ninguna tendencia.

Después de cumplir la etapa en el invernadero, las estacas fueron transplantadas al vivero. Transcurrido un año se evaluó la supervivencia y crecimiento que tuvieron en ese período. Los valores medios de las variables analizadas se presentan en el Cuadro $\mathrm{N}^{\circ} 3$.

Las estacas de tepa tratadas en invernadero con 18 y $21^{\circ} \mathrm{C}$ presentaron mejor crecimiento y sobrevivencia en vivero que aquellas con $24^{\circ} \mathrm{C}$. Se puede apreciar que las plantas que tuvieron mejor desarrollo y supervivencia, fueron aquellas con las cuales se logró inducir un mejor sistema radicular en invernadero. Estas plantas con una mayor masa radicular tuvieron mejores condiciones para absorber agua y nutrientes del suelo, lo que les permitió tener mayor crecimiento y supervivencia.

En vivero las estacas de lingue tuvieron una supervivencia cercana al $50 \%$. Por efecto de la alta mortalidad y debido a que a algunas plantas se les secó el ápice, aparentemente se presenta un decrecimiento en la altura. A esto las plantas reaccionaron generando nuevos brotes. Aunque a un nivel muy bajo también se observó crecimiento diametral. 


\section{Cuadro $\mathrm{N}^{\circ} 3$}

\section{SOBREVIVENCIA Y CRECIMIENTO EN VIVERO DE LAS ESTACAS ARRAIGADAS EN FUNCION DE DIFERENTES TEMPERATURAS EN INVERNADERO}

\begin{tabular}{|c|c|c|c|c|c|c|}
\hline \multirow[t]{2}{*}{ Especie } & \multirow{2}{*}{$\begin{array}{c}\text { Tratamiento } \\
\left({ }^{\circ} \mathrm{C}\right)\end{array}$} & \multirow{2}{*}{$\begin{array}{c}\text { Supervivencia } \\
\text { (1) } \\
\text { (\%) }\end{array}$} & \multicolumn{2}{|c|}{$\begin{array}{c}\text { Altura } \\
\text { (1) }\end{array}$} & \multicolumn{2}{|c|}{$\begin{array}{c}\text { Diametro } \\
\text { (1) }\end{array}$} \\
\hline & & & $\begin{array}{c}\text { Inicial } \\
(\mathrm{cm})\end{array}$ & $\begin{array}{l}\text { Final } \\
(\mathrm{cm})\end{array}$ & $\begin{array}{c}\text { Inicial } \\
\text { (cm) }\end{array}$ & $\begin{array}{l}\text { Fina } \\
(\mathrm{cm})\end{array}$ \\
\hline Tepa & $\begin{array}{l}18 \\
21 \\
24\end{array}$ & $\begin{array}{l}80 a \\
89 a \\
50 b\end{array}$ & $\begin{array}{l}16,5 \mathrm{a} \\
14,1 \mathrm{~b} \\
13,3 \mathrm{~b}\end{array}$ & $\begin{array}{l}24,5 a \\
20,7 b \\
14,4 \mathrm{c}\end{array}$ & $\begin{array}{l}6,4 a \\
6,3 a \\
6,2 a\end{array}$ & $\begin{array}{l}8,2 \mathrm{a} \\
7,7 \mathrm{a} \\
7,0 \mathrm{a}\end{array}$ \\
\hline Lingue & $\begin{array}{l}18 \\
21 \\
24 \\
\end{array}$ & $\begin{array}{l}48 a \\
57 a \\
48 a \\
\end{array}$ & $\begin{array}{l}14,5 a \\
13,2 a \\
14,4 a \\
\end{array}$ & $\begin{array}{c}11,2 \mathrm{ab} \\
9,8 \mathrm{~b} \\
12,8 \mathrm{a} \\
\end{array}$ & $\begin{array}{l}3,9 a \\
3,9 a \\
3,9 a\end{array}$ & $\begin{array}{l}4,4 \mathrm{~b} \\
4,5 \mathrm{~b} \\
4,8 \mathrm{a} \\
\end{array}$ \\
\hline Mañio & $\begin{array}{l}18 \\
21 \\
24\end{array}$ & $\begin{array}{l}48 b \\
74 a \\
84 a\end{array}$ & $\begin{array}{l}9,0 \mathrm{a} \\
7,8 \mathrm{a} \\
8,4 \mathrm{a}\end{array}$ & $\begin{array}{r}11,7 a \\
9,4 a \\
10,7 a\end{array}$ & $\begin{array}{l}2,1 \mathrm{a} \\
2,3 a \\
2,3 a\end{array}$ & $\begin{array}{l}3,0 \mathrm{a} \\
3,0 \mathrm{a} \\
3,1 \mathrm{a}\end{array}$ \\
\hline
\end{tabular}

(1) a, b, c: Valores promedios señalados con letras minúsculas distintas se diferencian entre ellos a un nivel de confianza del $95 \%$.

Las estacas de mañío tratadas con 21 y $24^{\circ} \mathrm{C}$ tuvieron una alta supervivencia en vivero.Con estos tratamientos se logró una mayor producción de raíces y, al igual que para las estacas de tepa, estas plantas pudieron captar más agua y nutrientes. El crecimiento en altura y diámetro no fue significativo.

Se puede observar que las estacas con las cuales se logró producir un sistema radicular más desarrollado en invernadero, posteriormente en vivero tuvieron mejores condiciones para absorber agua y nutrientes, razón por la cual también tuvieron un mejor crecimiento y supervivencia.

\section{CONCLUSIONES Y RECOMENDACIONES}

Las conclusiones y recomendaciones que a continuación se presentan, corresponden a resultados que se estiman preliminares y que posteriormente deben ser ampliados y afinados.

La especie tepa se puede reproducir fácilmente a partir de estacas.

Para la producción de raíces no tiene importancia si las estacas de tepa son 
cosechadas en otoño o primavera, aun cuando la temperatura más adecuada podría ser diferente en uno y otro caso.

Una temperatura de $21^{\circ} \mathrm{C}$ en la base de las estacas de tepa colectadas en primavera resultó ser el tratamiento más adecuado para la inducción de raíces.

Lingue y mañío también se pueden reproducir vegetativamente a partir de estacas, pero tienen una mayor mortalidad y menor inducción de raíces que tepa.

No se observan diferencias significativas en la producción de raíces al aplicar temperaturas entre 18 y $24^{\circ} \mathrm{C}$ en la base de las estacas de tepa cosechadas en primavera, por lo cual debe ampliarse el rango de temperaturas probadas.

Para mañío la mejor inducción de raices se logró con un nivel de temperatura de $24^{\circ} \mathrm{C}$ en la base de las estacas colectadas en primavera.

Es recomendable estudiar y comparar plantaciones realizadas con plantas de tepa, lingue y mañío producidas en forma generativa y vegetativa.

\section{REFERENCIA BIBLIOGRAFICAS}

Krussman, G., 1981. Die Baumschule. Paul Prey - Berlin und Hamburg. 656 pp.

Santelices, R., 1990. Propagación vegetativa de Tepa (Laurelia philippiana) a partir de estacas. Revista Ciencia e Investigación Forestal 4 (1). INFOR-CORFO. Chile. 61 - 68 pp.

Silva, J., 1968. Arraigamiento de estacas de Rauli (Nothofagus alpina Poepp. et Endl Oerstd). Tesis Universidad de Chile. Fac. de Agronomia, Escuela de Ingenieria Forestal. Chile. $23 \mathrm{pp}$. 